\section{SP1-92 SERUM $\alpha$-LINOLENIC ACID AND DISABLING DEMENTIA AMONG JAPANESE: THE CIRCULATORY RISK IN COMMUNITIES STUDY (CIRCS)}

doi:10.1136/jech.2011.142976n.69

\begin{abstract}
${ }^{1,2} \mathrm{~K}$ Yamagishi, ${ }^{*}{ }^{1} \mathrm{C} \mathrm{L}$ Chei, ${ }^{3} \mathrm{~A}$ Ikeda, ${ }^{3} \mathrm{E}$ Eguchi, ${ }^{3} \mathrm{H}$ Noda, ${ }^{3} \mathrm{~T}$ Ohira, ${ }^{2} \mathrm{M}$ Kiyama, ${ }^{2} \mathrm{~A}$ Kitamura, ${ }^{2} \mathrm{Y}$ Ishikawa, ${ }^{3} \mathrm{H}$ Iso. ${ }^{1}$ University of Tsukuba, Tsukuba, Japan; ${ }^{2}$ Osaka Medical Center for Health Science and Promotion, Osaka, Japan; ${ }^{3}$ Osaka University, Suita, Japan
\end{abstract}

Background Information on the impact of fatty acid composition on dementia has not yet been elucidated.

Methods We performed a nested case-control study based on a cohort of approximately 12000 Japanese people from two communities in the CIRCS, aged 45-85 at baseline (1984-1994). Fatty acid compositions were measured for 350 dementia cases and 700 controls (age, sex, community and baseline-year matched). The subjects were followed-up from 1999 through 2008, and incident disabling dementia was defined as dependent individuals who had moderate to severe dementia-related behavioural disturbance and/or cognitive impairment. This criterion was previously validated with 5 -cog test (specificity $90 \%$, positive predictive value $71 \%$ ). The conditional OR and 95\% CI for disabling dementia was calculated according to one SD increment of each fatty acid with adjustment for body mass index, smoking status, alcohol consumption, systolic blood pressure, antihypertensive medication use, serum total cholesterol and diabetes.

Results Serum $\boldsymbol{\alpha}$-linolenic acids were inversely associated with disabling dementia ( $O R=0.82$ [0.70-0.95] for 1-SD increment). No associations were observed for other fatty acids: $O R=1.07$ [0.91-1.26] for saturated fatty acids, 0.99 [0.84-1.17] for monounsaturated fatty acids, 0.98 [0.83-1.15] for $n-6$ polyunsaturated fatty acids, 1.00 [0.87-1.16] for eicosapentaenoic acid, and 1.03 [0.87-1.22] for docosahexaenoic acid

Conclusions We found a significant inverse association between serum $\alpha$-linolenic acid and incident disabling dementia.

\section{SP1-93 AN EPIDEMIOLOGICAL STUDY TO MEASURE THE PREVALENCE OF DIABETES IN THE URBAN AREAS AHMEDABAD, INDIA}

doi:10.1136/jech.2011.142976n.70

${ }^{1} \mathrm{H}$ Nayak, ${ }^{2} \mathrm{~S}$ Vyas, ${ }^{2} \mathrm{U}$ Oja, ${ }^{2} \mathrm{H}$ tiwari, ${ }^{2} \mathrm{~S}$ Parikh, ${ }^{2} \mathrm{~A}$ Solanki. ${ }^{1}$ B.J. Medical College, Ahmedabad, India; ${ }^{2}$ Smt.N.H.L.MMC, Ahmedabad, India

Introduction Diabetes is common, chronic, disproportionately expensive disorder showing secular trends. WHO predicted that India will have the highest number of diabetics in 2025. So present study was done to To know the prevalence of diabetes, to study the socio- demographic profile and Risk factors of diabetes among study population

Methods Current study was carried out in all the six zones of urban Ahmedabad. Required sample (900) to know the prevalence was stratified and one ward was selected from each zone. Information was collected from persons 20 year and above in standard predesigned and pretested proforma by house-to-house survey and blood for fasting glucose was collected on next day morning.

Results Out of 904, 125 (13.8\%) people were diabetics. $25(2.8 \%)$ people were newly diagnosed, while 100 (11.0\%) already had diabetes. The sex specific prevalence was $16.86 \%$ and $11.11 \%$ for males and females respectively. Prevalence of IFG was $6.0 \%(6.1 \%$ for males and $5.9 \%$ for females). The ratio of male \& female with diabetes was $1.4: 1 \quad(Z=2.51, p<0.05)$. Mean age of IFG was $44.61 \pm 14.44$, while the mean age of diabetics was $56.99 \pm 12.82$ $(Z=5.5, p<0.001)$. Overweight, Central obesity, SBP, DBP, Hypertension, Family history of diabetes, lack of physical activity at workplace, Sedentary occupation were found to be major risk factors for diabetes.

Conclusions Prevalence rate of diabetes among the study population was $13.8 \%$ and Prevalence of IFG was $6.0 \%$ which is alarming in this region and policy makers must take it seriously. Difference of around 10-20 years was found between IFG and Diabetes.

\section{SP1-94 DEPRESSION IN BRAZILIAN MUNICIPAL RESIDENTS WHOSE CULTURE OF TOBACCO IS A SIGNIFICANT ECONOMIC ACTIVITY}

doi:10.1136/jech.2011.142976n.71

U Otero, * J Chrisman, M C Câmara, P Brito, P Boccolini, P Guimarães. National Cancer Institute, Rio de Janeiro, Brazil

Introduction Brazil is the second-largest producer and largest exporter of tobacco in the world. The majority of tobacco production occurs in the southern states, where there is a predominance of family farming. Previous studies point to the excessive use of pesticides used during the culture of tobacco and health effects, including depression. The aim of this study was to estimate the prevalence of depression in a city where there is cultivation of tobacco.

Methods The work was conducted by the National Cancer Institute, in Paraíso do Sul municipality, southern Brazil, between October and December 2007. We selected men and women over 18 years $(N=2044)$. A questionnaire was applied, which sought to characterise their socioeconomic conditions, health and agricultural work. For this study, in addition to depression (the outcome) the following were selected as covariates age, sex, tobacco use, alcohol dependence and exposure to pesticides.

Results The prevalence of depression was $25 \%$ in women and $11 \%$ in men. When stratified by age categories, it was $27.1 \%, 42.4 \%$ and $34.1 \%$ in women aged $>29$ years, $30-49$ years and $>50$ years, respectively $(p=0.004)$. Among women agricultural workers, the prevalence was significantly greater in those directly exposed to pesticides (31\%) compared to those not directly exposed $(19 \%)$ $(\mathrm{p}=0.01)$. The prevalence (both sexes) was higher among those with alcoholism $(42.9 \%)$, both suspected cases $(33.7 \%)$ and undetected cases $(18.9 \%)(p=0.002)$.

Conclusion The prevalence of depression in Paraíso do Sul was higher than other southern regions in Brazil (7\%). Additional studies are needed to further investigate this outcome, especially in women involved in the cultivation of tobacco.

\section{SP1-95 TYPE 2 DIABETES MELLITUS AND MALIGNANT NEOPLASM: IS THERE ASSOCIATION?}

doi:10.1136/jech.2011.142976n.72

L Mello, ${ }^{*}$ A Nunes, L Franco. Faculty of Medicine of Ribeirao Preto-University of Sao Paulo, Ribeirao Preto/Sao Paulo, Brazil

Background Type 2 Diabetes Mellitus (T2DM) and cancer are diseases of increasing prevalence whose complications are related to decreased productivity, quality of life and survival. Recent studies suggest association of type 2 diabetes mellitus with cancer of breast, colon, prostate and others. Early diagnosis and proper treatment of these diseases could minimise the high costs for the public health as well as prevent severe complications and sequelae.

Methods Secondary information about hospital admissions in 34 hospitals of Ribeirao Preto/Brazil and 26 other cities were obtained from records of the Center for Data Processing linked to the Department of Social Medicine of Faculty of Medicine of Ribeirao Preto/University of Sao Paulo. The study was approved by the Ethics Committee of HCFMRP/USP. 